\title{
Real-time Construction of 3D Welding Torch in Virtual Space for Welding Training Simulator
}

\author{
Fangming, Yuan ${ }^{\mathrm{a} 1}$ \\ ${ }^{a l}$ Wuhan University of Technology, Hu Bei Provence, Wu Han 430063, China
}

Received: 16 April 2019; Accepted: 11 August 2019; Published: 08 September 2019

\begin{abstract}
One unsolved problem in the development of an effective welding training simulator is how to construct an accurate 3D welding torch model based on the moving position of this torch in the training process. This paper presents an effective approach to deal with the problem. The whole scene is constructed in the base coordinate system and the torch is modeled as a $3 \mathrm{D}$ object in the sub-coordinate system. The sub-coordinate system firstly overlaps the base coordinate system, and it's continuously changing as the trainer operates the torch. A nine axis sensor is installed in the torch at a selected point, which is the origin of the sub-coordinate system. The sensor can measure the rotation posture of the torch. Another marked point that can be captured by the Binocular Vision System (BVS) is installed with an infrared emitter. The BVS can measure the coordinate values of this point in the base coordinate system. As long as the coordinate values of a certain point on the model and its rotation posture based on this point can be determined, the VR development tool, such as Unity-3d,can track the model in real-time. That is the algorithm of this system, which is verified by Pro/E, a 3D modeling software. The approach presented above is applied to a welding training simulator product, which has been put into use and proved to be effective.
\end{abstract}

Index Terms: Virtual and Augmented Reality Applications, Welding simulator, Real-time positioning system, Real-time visualization.

(C) 2019 Published by MECS Publisher. Selection and/or peer review under responsibility of the Research Association of Mode rn Education and Computer Science

\footnotetext{
* Corresponding author.

E-mail address: fangming1995@163.com
} 


\section{Introduction}

In practical production, welding is widely used, from bridge construction, space shuttle, large ships to building materials, bicycle production and door manufacturing. The types of welding are classified according to different standards, and most of them are done by human workers. Therefore, the quality and efficiency of welding largely depend on workers' operating skills and experience. However, the improvement of workers' operating skills can only be achieved through multiple welding practices. Argon arc welding, for example, produces a lot of smoke and strong light, and the workers have to wear heavy protective equipment. Welding gun and welding rod in the actual operation process, on the other hand, will consume a lot of human and material resources, so there are great drawbacks and defects in the actual welding operation as a way of training workers. The purpose of this study is to design an efficient and environmentally friendly virtual welding simulator, which can be used by workers to replace the actual welding gun for welding training.

Using virtual welding training technology instead of physical welding for welding training has incomparable advantages, but the development of such virtual welding system contains many technical difficulties. One of them is how to locate the virtual welding torch and determine the pose of the torch in real time. Many researchers have made great efforts in this field.

On the basis of summarizing predecessors' work, we put forward a new real-time positioning algorithm for welding gun. This algorithm has been strictly calculated and proved, and the solid model is established in PRO/E software, which verifies the correctness and accuracy of this algorithm. In the section 2, we list the relevant researches in this field, and learn the excellent part and successful experience from these researches.

In section 3, the hardware structure and working principle of the virtual welding gun simulator are described. Compared with text, graphic representation has more intuitive advantages. In section 4, we introduce the important hardware components, and introduce the principle of Nine-axis sensor and Binocular Vision System in detail.

In section 5, the real-time positioning algorithm of the virtual welding simulator is described in detail, including matrix operation process and applied mathematical equations. In the section 6 , the algorithm described above is verified by $3 \mathrm{D}$ modeling software, and the practical application of the virtual welding simulator is introduced.

At the end of the article, the advantages of the algorithm and the current limitations are summarized and discuss the prospect for the future work.

\section{Literature review}

Virtual reality technology (VR) has been developed for a long time, with a perfect knowledge system and research methods. This technology has a wide range of applications, which can be integrated with many practical working scenes. The application of virtual reality technology in welding scene is a big promotion of virtual reality technology, and it also overcomes the unbearable difficulties in actual welding scene, such as high temperature, gas, strong light and so on. Up to now, many scholars and research institutions have conducted comprehensive researches in this field and achieved good results.

Kenneth Fast built a virtual welding system to simulate the gas metal arc welding (GMAW) process. The system makes use of a real GMAW welding torch, which is connected to a 3 DOF haptic device. The haptic device has a work envelope of $1 \mathrm{~m}^{3}$ and a force resolution of $1 \mathrm{gm}$. Translational forces are applied at the tip of the welding torch. The motion of the welding torch is tracked by using a commercial inertial 6 DOF tracking system and the angular orientation of the welding torch is also tracked by three-axis measurement gimbals. The real-time tracking data is used to update the force model for the haptic device and viewpoint for the visual display [1]. 
Scholar YiZhong Wang and his team developed a simulation model to simulate the wet arc welding, which is operated underwater. A haptic underwater wet arc welding training system is established, which mainly consists of three parts: a haptic device, an underwater wet arc welding model, and a virtual welder diver training model. The underwater wet arc welding model is used to generate proper underwater wet welding parameters for different wet welding situations. The virtual welder diver training model receives the welder diver's performance data, evaluates the performance, and generates the feedback to correct the errors of wet welding [2] .

Steven White and his teammates developed a welding training system that can simulate the welding process in real-time and give feedback to welders and the feedback can be used to analyze the process by the teacher afterwards. The system is based mainly on COTS components, a standard PC with a Dual-core CPU, and a medium-end nVidia graphics card. The torch is tracked by an Opti-Track system with 3 FLEX: V100 cameras. The same is also used to track a regular welding helmet to get accurate eye positions for display. The display itself is a Zalman Trimon stereo monitor that is laid out horizontally[3].

In the study of Yukang Liu, he designed a system that can simulate the GTAW process. The system consists of two workstations: welding station and virtual station. The welding station consists of an industrial welding robot, an eye view camera, and a compact 3D weld pool sensing system. In the virtual station a human welder can view the mock up where the weld pool image is rendered and displayed, and moves the virtual welding torch accordingly as if the operator is right in front of the work-piece. The human welder's movement is accurately captured by a Leap motion sensor, and the obtained virtual welding torch tip 3D coordinates will be sent to the PC.

These researches mentioned above provide several methods of the simulation system, but most of the authors didn't illustrate the algorithm of determining the location and pose of the torch in real time. That algorithm is the key point of this design, and it is essential to demonstrate the method and the algorithm clearly.

The difficulty of this design is to determine the coordinate values of a certain point on the welding torch and the rotation posture of the torch based on that point. In order to accurately locate the simulated welding torch in real time, we set the installation point of the nine-axis sensor as the reference point. In this paper, binocular vision system and nine-axis sensor are adopted to the system. The coordinate values of the infrared marked point can be obtained by the BVS, and the coordinate values of the nine-axis sensor can be deduced accurately from this and the transformation matrices. With the rotation angles being measured by the nine-axis sensor, the goal of real-time positioning of the simulated welding torch can be achieved. It has been verified that the algorithm is accurate and the model can provide a good man-computer interaction experience. This method can be applied to practical welding training products that can be put into mass production.

\section{System framework}

The hardware structure of this system is shown in the Fig.1.

The design of hardware components includes main control computer, image acquisition card, cameras, the simulated welding torch, test plate and the monitor. The motion parameters are measured by a nine-axis sensor, and the binocular vision system is used to determine the position coordinates of the marked point in the welding torch.

The flowchart of the system is shown in Fig.2. This flowchart intuitively shows the working process of the virtual welding simulator. Firstly, the position of the welding gun is measured by the Binocular Vision System, which is represented by the coordinate value in the global coordinate system, Then the Nine-axis sensor measures the real-time tilt angle of the welding gun, and the computer determines the real-time position of the welding gun by integrating the coordinate value of the welding gun and the tilt angle. 
Dual cameras measure welding torch in real time

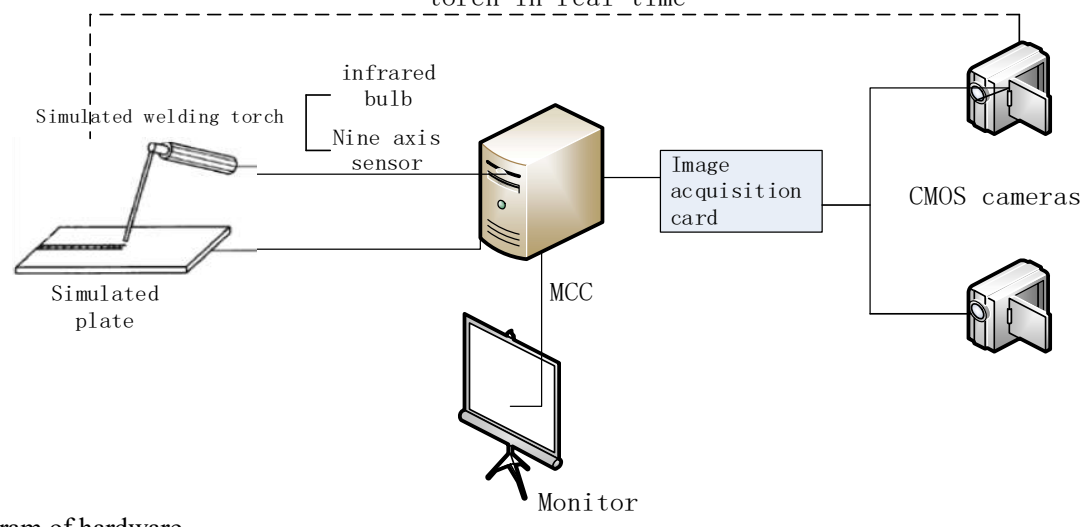

Fig. 1. Structure diagram of hardware

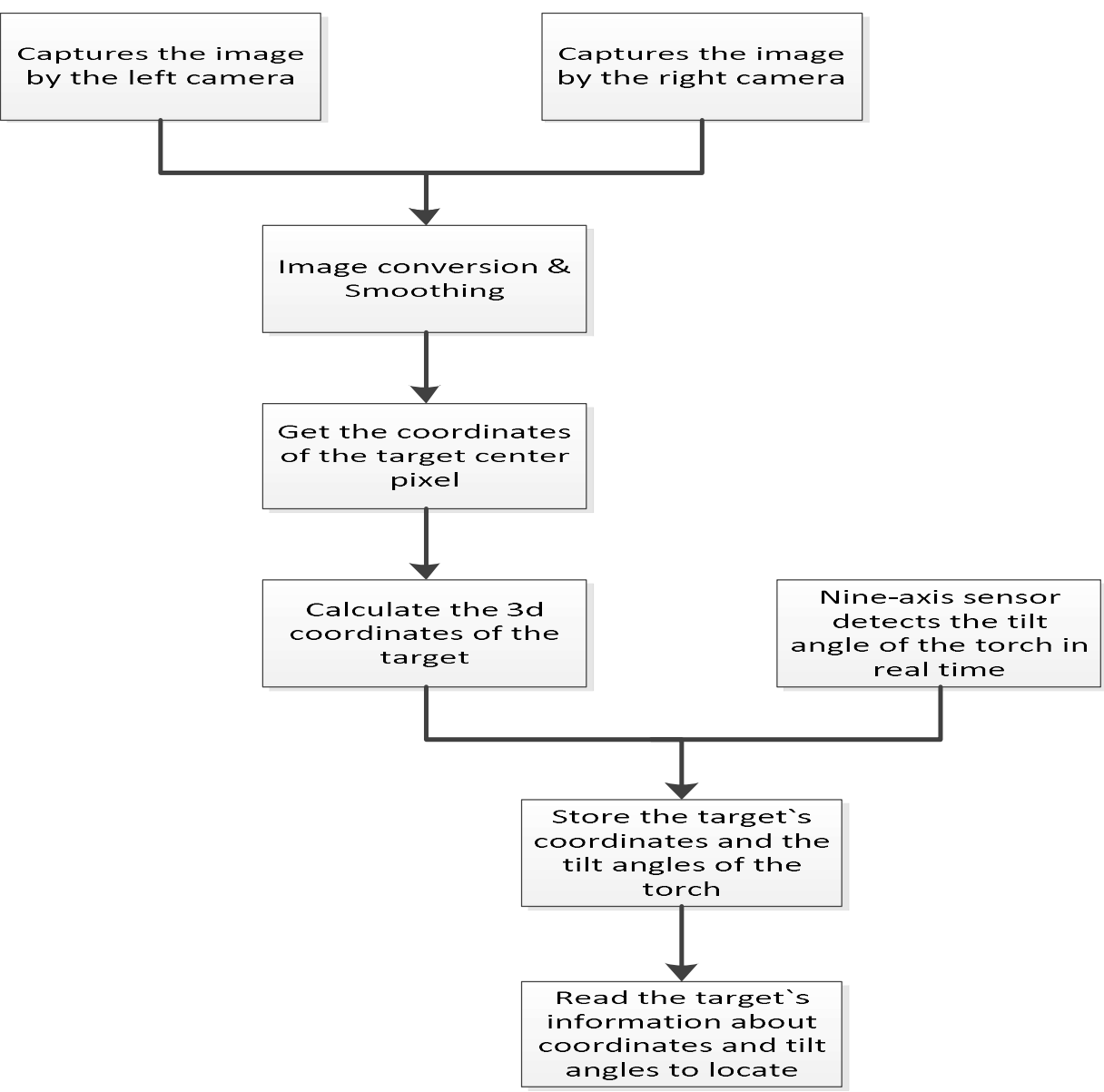

Fig.2. Flowchart of this system 


\section{Measuring equipment}

\subsection{Nine axis sensor}

The nine-axis sensor is a combination of three sensors: a three-axis accelerometer, a three-axis gyroscope, and a three-axis magnetometer. The three-axis accelerometer measures the acceleration of the object by indirectly measuring the force on the object, and presents the motion of the object. The three-axis gyroscope mainly measures the rotation rate of the object on $\mathrm{X}, \mathrm{Y}$ and $\mathrm{Z}$ axes, that is, the rate of changes of the rotation angles; By measuring the intensity and direction of the magnetic field, the three-axis magnetometer locates the orientation of the object and measures the angles between the object and each coordinate axis. Nine-axis sensor is an integrated sensor module, which reduces the circuit board and overall space. Compared with other sensors, nine-axis sensor is more suitable for light and portable electronic devices and wearable products, with better measurement accuracy and function.

The data accuracy of the integrated sensor depends on the accuracy of the equipment itself, and also involves the correction after welding and assembly, as well as the matching algorithm for different usage scenarios. The motion state of the object can be determined according to the data measured by the accelerometer. The suitable algorithm fuses the data from various sensors to make up for the shortcomings of single sensor in calculating the precise orientation and direction. So the nine-axis sensor can achieve high precision motion detection. Therefore, nine-axis sensor is the most suitable measuring equipment in a welding simulator. By working together, the nine-axis sensor calculates the real-time position of the object in the help of the fusion algorithm. (Fig.3.)

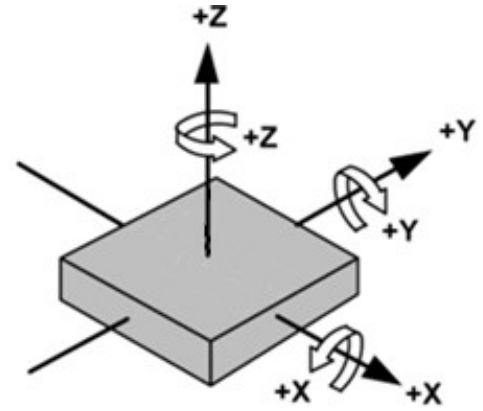

Fig.3(a-b). Schematic diagram of nine-axis sensor

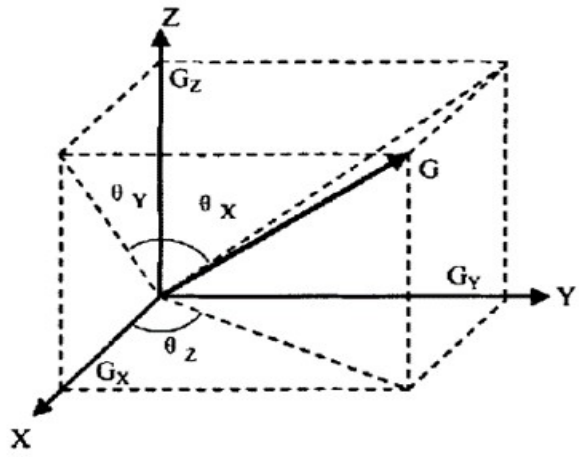

\subsection{Binocular Vision System}

The Binocular Vision System is an important form of machine Vision. The working principle of Binocular Vision System is based on the principle of human vision, and it's a way for computers to perceive distance. By observing an object from two or more points, images from different perspectives can be obtained. According to the matching relationship of pixels between images, the offset between pixels can be calculated based on the triangulation principle, so as to obtain the $3 \mathrm{~d}$ information of objects. With the depth of field information of the object, we can calculate the actual distance between the object and the camera, the $3 \mathrm{~d}$ size of the object, and the actual distance between two points. 


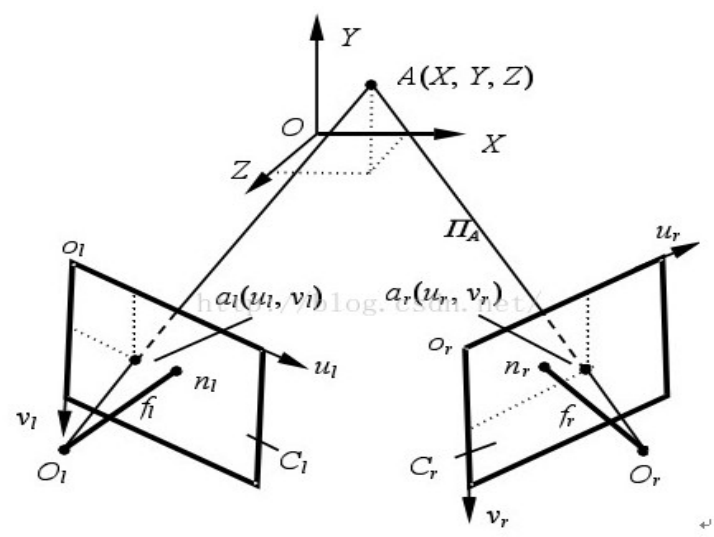

Fig.4. Schematic diagram of binocular vision system

In order to accurately obtain the depth information of a certain point in $3 \mathrm{~d}$ space, we need to obtain parameters such as focal length $\mathrm{f}$, parallax $\mathrm{d}$ and camera center distance $T_{x}$. To get the $\mathrm{X}$ and $\mathrm{Y}$ coordinates of a certain point, you also need to know the deviation of the left and right plane coordinate system and the central point of the stereo coordinate system, $C_{x}$ and $C_{y}$. Therefore, we need to carry out the following three steps: camera calibration, image correction and stereo matching. Due to the limited space of the article, the detailed introduction of these three steps is omitted here.

The realization of binocular stereoscopic imaging is based on the principle of parallax, and its model is shown in the figure. This model is based on a set of mathematical models that are non-distortion, alignment and based on a measured standard stereoscopic experimental table. The stereo vision system is composed of two cameras on the right and left. The coordinate values on the imaging plane $C_{l}$ and $C_{r}$ in the two cameras of point $\mathrm{A}(\mathrm{X}, \mathrm{Y}, \mathrm{Z})$ are $a_{l}\left(u_{l}, v_{l}\right)$ and $a_{r}\left(u_{r}, v_{r}\right)$, respectively. These two image points are the images of the same object point A, which are called "Conjugate Points". Making the connections respectively between them and the optical centers of the two cameras $O_{l}$ and $O_{r}$, the intersection point is the object $\mathrm{A}(\mathrm{X}, \mathrm{Y}, \mathrm{Z})$ in the world space. That's the basic principle of stereo vision. (Fig.4.)

\section{Algorithm}

In this positioning system, the coordinate systems are all right-handed. After determining the world coordinate system, the welding torch coordinate system is established on this basis. There is an infrared emitter point $\mathrm{B}$ on the simulated torch, whose coordinate values $\left(X_{B}, Y_{B}, Z_{B}\right)$ in the world coordinate system are measured by the binocular vision system. A nine axis sensor is installed in the torch at a selected point $\mathrm{C}$, which is the origin of the welding torch coordinate system. The nine-axis sensor measures the tilt angles of the welding torch $(\alpha, \beta, \gamma)$ in real time. With the help of the transformation matrix between coordinate systems, the coordinate values of point $\mathrm{C}$ in the world coordinate system and the real-time rotation posture of the welding torch are derived from the known coordinate values of point $\mathrm{B}$ and the tilt angles of the welding torch. (The model diagram is shown in the Fig.5.) 


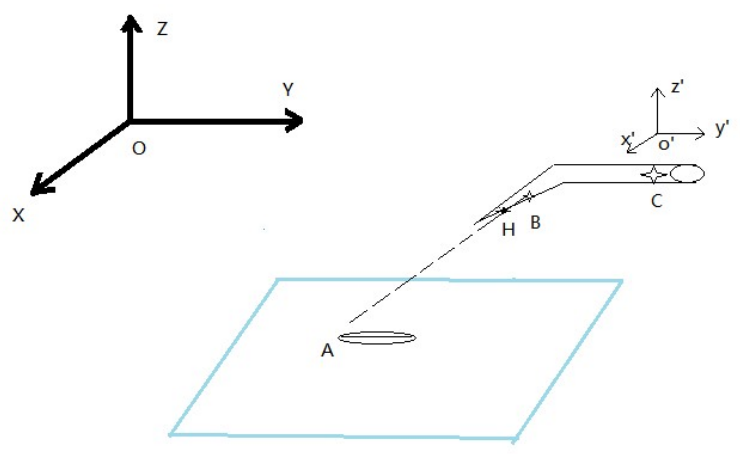

Fig.5. Model diagram of simulator

There are three types of rotation matrices of coordinate system about the $\mathrm{X}$ axis, $\mathrm{Y}$ axis and $\mathrm{Z}$ axis. The rotation matrix is shown as follows:

$$
R_{Z}=\left[\begin{array}{ccc}
\cos \gamma & -\sin \gamma & 0 \\
\sin \gamma & \cos \gamma & 0 \\
0 & 0 & 1
\end{array}\right] ; R_{X}=\left[\begin{array}{ccc}
1 & 0 & 0 \\
0 & \cos \alpha & -\sin \alpha \\
0 & \sin \alpha & \cos \alpha
\end{array}\right] ; R_{Y}=\left[\begin{array}{ccc}
\cos \beta & 0 & \sin \beta \\
0 & 1 & 0 \\
-\sin \beta & 0 & \cos \beta
\end{array}\right]
$$

In the initial state, the infrared emitter's coordinates in the torch coordinate system are known as B $\left(X_{B}^{t}, Y_{B}^{t}, Z_{B}^{t}\right)$. The coordinates of point $\mathrm{C}$ on the welding torch in the world coordinate system $\left(X_{C}, Y_{C}\right.$, $Z_{C}$ ) are the values to be solved. The rotation matrices are combined in a certain order to obtain the rotation transformation matrix. In this paper, coordinate values of B are calculated in accordance with the commonly used rotation order of $\mathrm{z}-\mathrm{x}-\mathrm{y}$ axis. The coordinate relation of point $\mathrm{B}$ between the two coordinate systems is shown as follows:

$$
\left[\begin{array}{c}
X_{B} \\
Y_{B} \\
Z_{B}
\end{array}\right]=\left[\begin{array}{c}
X_{c} \\
Y_{C} \\
Z_{c}
\end{array}\right]+\left[\begin{array}{c}
X_{B}^{v r} \\
Y_{B}^{H} \\
Z_{B}^{H}
\end{array}\right]
$$

$$
\left[\begin{array}{c}
X_{B}^{\prime} \\
Y_{B}^{\prime} \\
Z_{B}^{\prime}
\end{array}\right]=R_{Y}{ }^{*} R_{X}{ }^{*} R_{Z}\left[\begin{array}{c}
X_{B}^{\prime \prime} \\
Y_{B}^{\prime \prime} \\
Z_{B}^{\prime \prime}
\end{array}\right]
$$

Integrate equations (2) and (3), and we can get:

$$
\begin{aligned}
& {\left[\begin{array}{l}
X_{c} \\
Y_{c} \\
Z_{c}
\end{array}\right]=\left[\begin{array}{l}
X_{B} \\
Y_{B} \\
Z_{B}
\end{array}\right]-\left[R_{Y}{ }^{*} R_{X}{ }^{*} R_{Z}\right]^{-1}\left[\begin{array}{c}
X_{B}^{\prime} \\
Y_{B}^{\prime} \\
Z_{B}^{\prime}
\end{array}\right]} \\
& {\left[\begin{array}{l}
X_{c} \\
Y_{C} \\
Z_{c}
\end{array}\right]=\left[\begin{array}{c}
X_{B} \\
Y_{B} \\
Z_{B}
\end{array}\right]-\left[\begin{array}{ccc}
\cos \beta \cos \gamma-\sin \beta \sin \alpha \sin \gamma & -\cos \alpha \sin \gamma & \sin \beta \cos \gamma+\sin \alpha \cos \beta \sin \gamma \\
\cos \beta \sin \gamma+\sin \beta \sin \alpha \cos \gamma & \cos \alpha \cos \gamma & \sin \beta \sin \gamma-\sin \alpha \cos \beta \cos \gamma \\
-\sin \beta \cos \alpha & \sin \alpha & \cos \alpha \cos \beta
\end{array}\right] \cdot\left[\begin{array}{c}
X_{B}^{\prime} \\
Y_{B}^{\prime} \\
Z_{B}^{r}
\end{array}\right]}
\end{aligned}
$$

According to the above formula, the coordinate values of point $\mathrm{C}$ in the world coordinate system can be obtained. Since the rotation matrix is an orthogonal matrix, its inverse matrix is the transposed matrix of the original matrix, and that's where the rotation matrix comes in handy. $\left(X_{E}^{t}, Y_{E}^{t}, Z_{E}^{t}\right)$ represents the positional relationship between point $\mathrm{B}$ and point $\mathrm{C}$ in the initial situation. This value is accurately determined when constructing the torch model and is one of the known parameters of the system. $\left(X_{E}^{i t}, Y_{E}^{u}, Z_{E}^{u s}\right)$ is an intermediate variable that represents the positional relationship between point $\mathrm{B}$ and point $\mathrm{C}$ at any time, that is, the coordinate value of point B in the torch coordinate system at any time. 
After finding the coordinate values of point $\mathrm{C}$ in the world coordinate system, set a point $\mathrm{H}$ at the end of the welding torch, then connect B、H and extend the linear. The intersection of linear BH with X-O-Y plane of the world coordinate system is the location of A, the welding molten pool. According to the spatial linear equation:

$$
\frac{X-X_{1}}{X_{1}-X_{2}}=\frac{Y-Y_{1}}{Y_{1}-Y_{2}}=\frac{Z-Z_{1}}{Z_{1}-Z_{2}}
$$

Point $\mathrm{A}$ is on the $\mathrm{X}-\mathrm{O}-\mathrm{Y}$ plane (Seen in the Fig.5.), therefore $Z_{A}$ equals 0 . Then we can get the coordinate values of the welding molten pool in the world coordinate system $\left(X_{A}, Y_{A}, Z_{A}\right)$.

\section{Examination and field application}

\subsection{Examination}

In this paper, the Pro/E 3d modeling software is used to build the models and to verify the correctness of coordinate algorithm. Firstly, the point $\mathrm{C}$ on the nine-axis sensor of the welding torch is selected to set up the $3 \mathrm{D}$ model of the welding torch. Then set the positional relationship between the infrared emitter point $\mathrm{B}$ and the nine axis sensor installation point $\mathrm{C}$. It means that $\left(X_{E}^{t}, Y_{E}^{t}, Z_{E}^{t}\right)$ has been determined.

Operate the software to rotate the model around the three axis for $\boldsymbol{\alpha} 、 \boldsymbol{\beta} 、 \boldsymbol{\gamma}$ respectively, and thus simulate the welding torch in new position and posture in the space. To make it convenient and clear, set the infrared emitter's initial coordinate values $\left(X_{B}^{s}, Y_{B}^{\prime}, Z_{B}^{\prime}\right)$ in the torch coordinate system as $(-50,-50,0)$ and point $\mathrm{C}$ in the world coordinate system $\left(X_{c}, Y_{c}, Z_{c}\right)$ for $(100,100,0)$

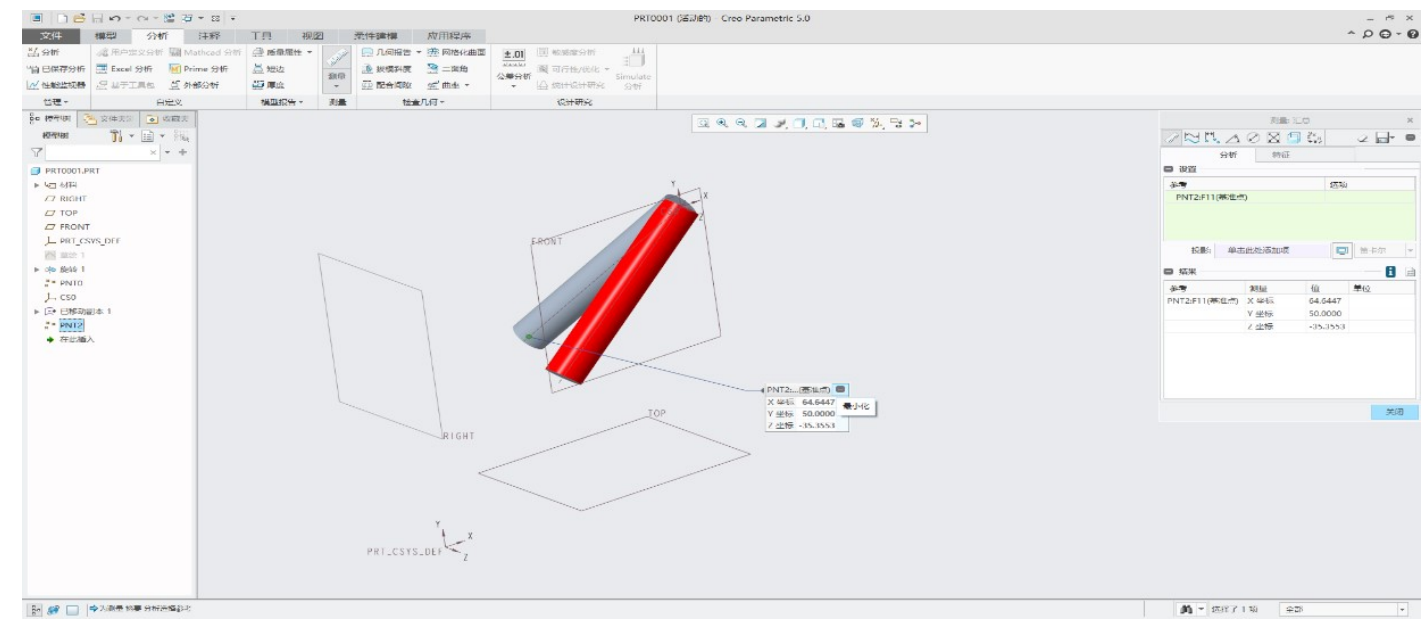

Fig.6.The interface of model test in the software

There are three groups of model verification processes. As is shown in Table 1, the model is rotated around the coordinate axis according to the values in the table. Then the coordinates of point B in the base coordinate system are measured. By comparing the coordinate values calculated by the above algorithm and the measured coordinate values, the correctness of the algorithm is obtained. Fig.6. shows the interface of the Pro/E software model validation. In this software the coordinate values of the marker on the model can be displayed directly. The red model in Fig.6 represents the position before the rotation transformation, and the gray model represents the position after the rotation transformation. 
By measuring the position coordinates of point $\mathrm{B}$ in the base coordinate system $\left(X_{B}, Y_{B}, Z_{B}\right)$ and comparing them with the coordinate values calculated by the algorithm, the two sets of values are equal and the correctness of the algorithm is verified.

Table 1.The coordinate value of B in the base coordinate system

\begin{tabular}{|c|c|c|c|}
\hline The coordinate values after rotation & $\mathbf{X}$ & $\mathbf{Y}$ & $\mathbf{Z}$ \\
\hline The first group $\left(37^{\circ}, 45^{\circ}, 90^{\circ}\right)$ & 50.48 & 50.48 & 9.84 \\
\hline The second group $\left(45^{\circ}, 45^{\circ}, 45^{\circ}\right)$ & 64.64 & 50.0 & -35.36 \\
\hline The third group $\left(30^{\circ}, 30^{\circ}, 30^{\circ}\right)$ & 67.07 & 40.85 & 20.42 \\
\hline
\end{tabular}

\subsection{Field application}

The real-time positioning method designed in this paper has been successfully applied to practical products. Wuhan KeMa co. developed a new generation of welding simulator based on this positioning algorithm. Compared with the previous generation of products, this simulator has a limited improvement effect in terms of time delay reduction, etc., but the positioning accuracy of welding gun has been greatly improved. Therefore, the users can get a better human-computer interaction experience. In the process of further improvement, the trainer can get more intuitive feedback by adding voice prompts and enhancing the rendering effect.

The figure below shows the picture displayed when the welding simulator is working (Fig.7.).

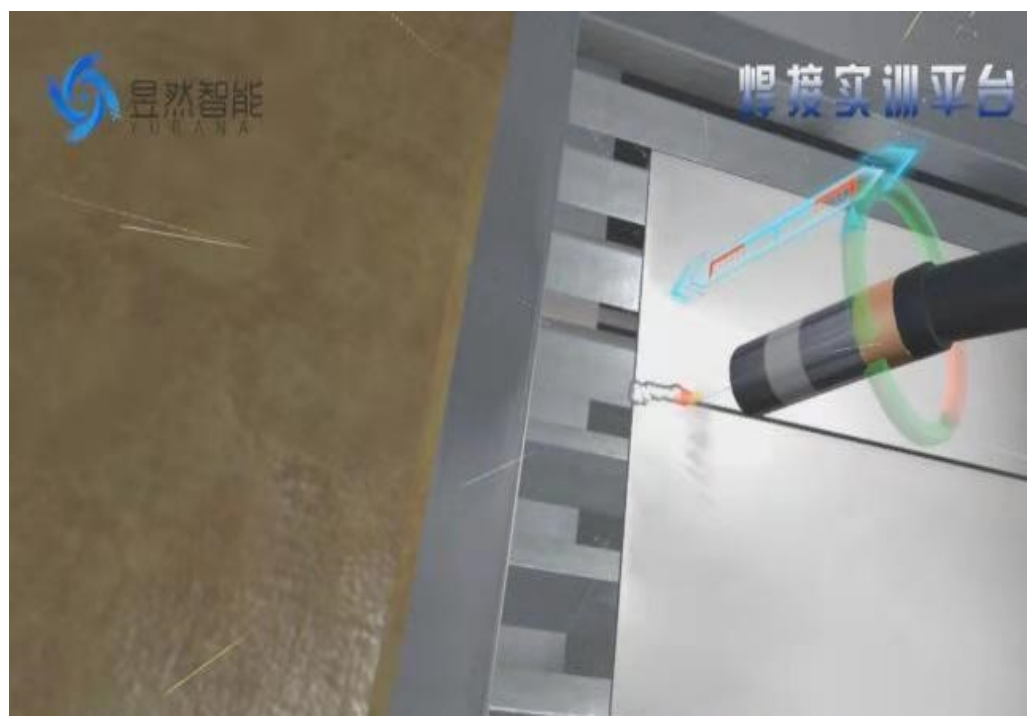

Fig.7. Operation interface of the simulator 


\section{Conclusions}

This paper introduces a real-time positioning system of virtual welding torch in the simulation welding training. Its basic principle is to obtain the exact coordinate values of unknown points according to the transformation matrix relation between the coordinate values of two spatial points. Based on this principle, a practical algorithm is designed to track the position and pose of the virtual welding torch. The accuracy of the algorithm is fully verified by comparing the results of several examples in 3D modeling tools.

Obtaining the accurate position information of the nine-axis sensor is the key to accurately positioning the virtual torch. If the installation point of the Nine-axis sensor and the infrared marker of the BVS are the same point, this problem will be easily solved. But in fact, these two points cannot be completely overlapped, because in the manufacturing process, this is impossible to achieve. Prior to this, most of the welding simulators placed the two points very closed, so they were treated as the same point. This method is inherently wrong, let alone its accuracy.

The positioning method designed in this paper has undergone rigorous derivation calculation, and it has solved the above mentioned shortcomings well, which greatly improves the positioning accuracy. However, there are also some limitations. For example, there will be further requirements for the welding torch production process to ensure the accuracy of their positional relationship. In addition, the production cost of the welding simulator will be a little higher. Looking ahead to the future work, the details of the welding simulator can be further improved and developed, so the users shall have a more vivid human-computer interaction experience. And we can figure out some ways to improve the service life of the welding simulation.

\section{Acknowledgements}

In the process of writing this article, Professor Guo Xing and Wang Guoxian have given me great help and recommended many references. I also want to thank my classmates for helping me solve many practical problems with the modeling software. Thank you, Ms. Zhu FeiFei, for helping me correct the grammatical errors in my manuscript. I would like to express my heartfelt thanks to the above friends.

\section{References}

[1] Fast, K., Gifford, T., Yancey, R. Virtual training for welding [P]. Mixed and Augmented Reality, 2004. ISMAR 2004. Third IEEE and ACM International Symposium on, 2004.

[2] Yizhong Wang, Yonghua Chen, Wenjie Zhang, Dingcheng Liu, Huafang Huang. Study on underwater wet arc welding training with haptic device [P]. VECIMS '09. IEEE International Conference on, 2009.

[3] White, S., Prachyabrued, M., Baghi, D., Aglawe, A., Reiners, D., Borst, C., Chambers, T. Virtual Welder Trainer [P]. Virtual Reality Conference, 2009. VR 2009. IEEE, 2009.

[4] Xiwen Liu. Single neuron self-tuning PID control for welding molten pool depth [P]. Intelligent Control and Automation, 2008. WCICA 2008. 7th World Congress on, 2008.

[5] Xin Yin, Zhen Zhang. Defect recognized system of friction welding based on compensatory fuzzy neural network [P]. Machine Learning and Cybernetics, 2009 International Conference on, 2009.

[6] Ning Huang,YuKang Liu,Shujun Chen,YuMing Zhang. Control of human welder's arm movement in (GTAW) process [P]. Advanced Intelligent Mechatronics (AIM), 2014 IEEE/ASME International Conference on, 2014.

[7] B. Xie, Q. Zhou and L. Yu, "A real-time welding training system base on virtual reality," 2015 IEEE Virtual Reality (VR), Arles, 2015, pp. 309-310. 
[8] U. Yang, G. A. Lee, Y. Kim, D. Jo, J. Choi and K. Kim, "Virtual Reality Based Welding Training Simulator with 3D Multimodal Interaction," 2010 International Conference on Cyberworlds, Singapore, 2010, pp. 150-154.

[9] S. Nawrocki, L. Hao and X. Tang, "Modeling \& analysis of weld short faults of bar-wound propulsion IPM machine part II: Phase-to-phase short," 2011 IEEE Vehicle Power and Propulsion Conference, Chicago, IL, 2011, pp. 1-4.

[10] B. Hazel, E. Boudreault, J. Côté and S. Godin, "Robotic post-weld heat treatment for in situ repair of stainless steel turbine runners," Proceedings of the 2014 3rd International Conference on Applied Robotics for the Power Industry, Foz do Iguassu, 2014, pp. 1-6.

[11] H. Tokunaga, N. Matsuki, H. Sawada, T. Okano and Y. Furukawa, "A robot simulator for manufacturing tasks on a component-based software development and execution framework," (ISATP 2005). The 6th IEEE International Symposium on Assembly and Task Planning: From Nano to Macro Assembly and Manufacturing, 2005., Montreal, Que., 2005, pp. 162-167.

[12] E. E. M. Mohamed, T. A. Ahmed and M. A. Sayed, "Real-time simulation of position control for linear induction motor drives using cascaded sliding mode control," 2018 International Conference on Innovative Trends in Computer Engineering (ITCE), Aswan, 2018, pp. 386-391.

[13] W. Yao and L. Ma, "Research and Application of Indoor Positioning Method Based on Fixed Infrared Beacon," 2018 37th Chinese Control Conference (CCC), Wuhan, 2018, pp. 5375-5379.

[14] G. Cao, L. Lin, H. Qiu and J. F. Pan, "Design and analysis of a dSPACE-based position control system for a linear switched reluctance motor," 2009 3rd International Conference on Power Electronics Systems and Applications (PESA), Hong Kong, 2009, pp. 1-4.

[15] T. LI and H. ZHU, "Research on model control of binocular robot vision system," 2018 Chinese Automation Congress (CAC), Xi'an, China, 2018, pp. 1794-1797.

[16] P. Hu, X. Hao, J. Li, C. Cheng and A. Wang, "Design and Implementation of Binocular Vision System with an Adjustable Baseline and High Synchronization," 2018 IEEE 3rd International Conference on Image, Vision and Computing (ICIVC), Chongqing, 2018, pp. 566-570.

[17] F. Zhao and Z. Jiang, "A New Algorithm for Three-dimensional Construction Based on the Robot Binocular Stereo Vision System," 2012 4th International Conference on Intelligent Human-Machine Systems and Cybernetics, Nanchang, Jiangxi, 2012, pp. 302-305.

[18] G. Yang, W. Jin and T. Xu, "Design and implementation of infrared-binocular vision system for robot navigation," 2011 Chinese Control and Decision Conference (CCDC), Mianyang, 2011, pp. 4185-4188.

[19] J. Deng, J. Li, X. Zou and F. He, "A Test System of Binocular Vision of Picking Robot," 2010 International Conference on Measuring Technology and Mechatronics Automation, Changsha City, 2010, pp. 369-372.

[20] C. Ruan, X. Gu, Y. Li, G. Zhang, W. Wang and Z. Hou, "Base frame calibration for multi-robot cooperative grinding station by binocular vision," 2017 2nd International Conference on Robotics and Automation Engineering (ICRAE), Shanghai, 2017, pp. 115-120.

[21] Wang, XW (Wang, Xuewu).Three-dimensional vision-based sensing of GTAW: a review[J].International Journal of Advanced Manufacturing Technology,2014,Vol.72,No.1-4. 


\section{Author's Profile}

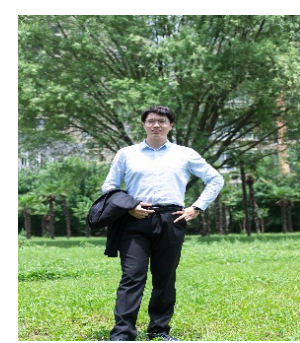

Fangming Yuan is currently a graduate student majoring in mechanical engineering at Wuhan University of Technology. He received his Bachelor degree in mechanical engineering from Wuhan University of Technology in July 2018. His fields of scientific interests are mechatronics and electrical intelligent control.

How to cite this paper: Fangming, Yuan. "Real-time Construction of 3D Welding Torch in Virtual Space for Welding Training Simulator", International Journal of Engineering and Manufacturing(IJEM), Vol.9, No.5, pp.34-45, 2019. DOI: 10.5815/ijem.2019.05.03 